\title{
Corrigendum: Drug resistance in eukaryotic microorganisms
}

Alan H. Fairlamb, Neil A. R. Gow, Keith R. Matthews and Andrew P. Waters

Nature Microbiology 1, 16092 (2016); published 24 June 2016; corrected 1 August 2016.

The version of this Review originally published incorrectly stated that artemisinin-resistance-associated mutation of KELCH13 results in its enhanced association with phosphatidylinositol-3-OH kinase $(\mathrm{PI}(3) \mathrm{K})$ when in fact association is reduced. All versions of the Review have been amended and the authors apologize for any confusion caused. 